\title{
(2) OPEN ACCESS \\ Association between hypotension and serious illness in the emergency department: an observational study
}

\author{
Nienke N Hagedoorn 두 , Joany M Zachariasse, Henriette A Moll
}

- Additional material is published online only. To view please visit the journal online (http://dx.doi.org/10.1136/ archdischild-2018-316231).

Department of Pediatrics, Erasmus MC-Sophia, Rotterdam, The Netherlands

\section{Correspondence to} Professor Henriette A Moll, Department of Pediatrics, Erasmus MC Sophia, Rotterdam 3000 CB, The Netherlands; h.a.moll@erasmusmc.nl

Received 17 September 2018 Revised 11 March 2019 Accepted 18 March 2019 Published Online First 4 April 2019

\section{Check for updates}

(c) Author(s) (or their employer(s)) 2020. Re-use permitted under CC BY. Published by BMJ.

To cite: Hagedoorn NN, Zachariasse JM, Moll HA Arch Dis Child

2020;105:545-551.

\section{ABSTRACT}

Background The value of routine blood pressure measurement in the emergency department (ED) is unclear.

Objective To determine the association between hypotension in addition to tachycardia and the Shock Index for serious illness.

Design Observational study.

Setting University ED (2009-2016).

Participants, methods and main outcomes Routine data collected from consecutive children $<16$ years. Using logistic regression, we assessed the association between hypotension (adjusted for tachycardia) and Shock Index (ratio heart rate/blood pressure [BP]) for serious illness. The predictive accuracy (sensitivity, specificity) for hypotension and Shock Index was determined for serious illness, defined as intensive care unit (ICU) and hospital admissions.

Results We included 10698 children with measured BP. According to three age-adjusted clinical cut-offs (Advanced Paediatric Life Support, Paediatric Advanced Life Support and Paediatric Early Warning Score), hypotension was significantly associated with ICU admission when adjusted for tachycardia (range OR 2.6-5.3). Hypotension showed low sensitivity (range 0.05-0.12) and high specificity (range 0.95-0.99) for ICU admission. Combining hypotension and tachycardia did not change the predictive value for ICU admission. Similar results were found for hospitalisation. Shock index was associated with serious illness. However, no specific cut-off value was identified in different age groups.

Conclusions Hypotension, adjusted for tachycardia, is associated with serious illness, although its sensitivity is limited. Shock index showed an association with serious illness, but no acceptable cut-off value could be identified. Routine BP measurement in all children to detect hypotension has limited value in the ED. Future studies need to confirm which patients could benefit from BP measurement.

\section{INTRODUCTION}

Vital signs are essential for recognising serious illness in children in the emergency department (ED). However, the frequency of blood pressure (BP) measurement varies widely $(23 \%-87 \%)$ and no consensus exists on performing routine $\mathrm{BP}$ measurement to detect hypotension. ${ }^{1-3}$ Accurate age-related cut-offs are needed to assess hypotension as incorrect cut-offs may lead to false-positive or false-negative results. Although paediatric guidelines provide different definitions of low BP, it is

\section{What is already known on this topic?}

- Hypotension is considered a late feature of serious illness in children and different reference values exist for hypotension.

- In the adult emergency department population, high Shock Index is associated with mortality, severity of illness and hospital admission.

\section{What this study adds?}

- Hypotension has additional value over tachycardia, but due to its low sensitivity clinical relevance is limited.

- High Shock Index is associated with serious illness in different age groups. Acceptable cutoff values could not be identified.

- Blood pressure measurement for detection of hypotension is suggested to be of limited value in all children attending the emergency department.

unclear which BP cut-off should be used in the ED. ${ }^{4-6}$

Moreover, the predictive value of hypotension for serious illness is unclear in the diverse ED population. In children, hypotension is considered a late sign of deterioration and is used for diagnosis of shock. Children increase heart rate to preserve cardiac output. ${ }^{78}$ Since abnormal heart rate occurs in an earlier phase, the additional value of routine $\mathrm{BP}$ over heart rate in prediction of serious illness could be limited in the ED.

Another measure of haemodynamic status is Shock Index, the ratio of heart rate to systolic $\mathrm{BP}$, which is associated with mortality and disease severity in adults. ${ }^{9-11}$ In small cohorts of children, elevated Shock Index has been associated with injury severity in trauma and mortality in septic shock. ${ }^{12-15}$ However, the Shock Index in all paediatric ED patients has not yet been evaluated and could be an important predictor in children.

This study aims to study the additional value of BP measurement: (1) To determine the predictive capability of hypotension in addition to tachycardia. (2) To assess the utility of Shock Index for serious illness in children. This observational study is based on routine $\mathrm{BP}$ measurements in the $\mathrm{ED}$ using electronic health records. 


\section{METHODS}

Design

We applied three commonly used clinical definitions for hypotension on data from a prospective study of children visiting the ED to determine the predictive value of hypotension in addition to tachycardia for serious illness. Second, we studied the predictive ability of the Shock Index. This was a secondary analysis in a study validating the Manchester Triage System (MTS). ${ }^{16} 17$

\section{Setting}

The observational study included all children $<16$ years who presented consecutively at the ED of Erasmus MC-Sophia Children's Hospital (Rotterdam, The Netherlands) between August 2009 and December 2016. This inner-city university hospital receives approximately 7000 children annually.

\section{Data collection}

Data of patient characteristics, vital signs, triage level and disposition were automatically derived from electronic health records that were completed by trained nurses during triage. Heart rate was measured using pulse oximeters and BP using the oscillometric infinity M540 monitor (Draeger Medical, Telford, Pennsylvania, USA). BP was measured on medical indication at the discretion of the nurse or attending physician.

\section{Outcomes and definitions}

Serious illness was defined as admission to the ICU or hospital following ED visit. Indications for ICU admission include requirement of advanced respiratory support ([non-] invasive ventilation, high flow oxygen); inotropes or continuous intravenous antiepileptics; tracheal cannula; acute or threatening failure of more than two organ systems which was expected to last $>24$ hours or in a child $<1$ year. ${ }^{17}$ We selected three ageadjusted clinical cut-offs to define hypotension to demonstrate the range in clinical practice: Advanced Paediatric Life Support (APLS) ${ }^{18}$ Paediatric Advanced Life Support (PALS)/septic shock screening tool ${ }^{19} 20$ and the Paediatric Early Warning Score ${ }^{21}$

\begin{tabular}{|c|c|c|c|}
\hline Age range & $\mathrm{APLS}^{18}$ & PEWS $^{34}$ & PALS $^{1920}$ \\
\hline$<4$ weeks & $<75$ & $\leq 60$ & $<60$ \\
\hline 4-6 weeks & $<75$ & $\leq 60$ & $<70$ \\
\hline 6 weeks to 3 months & $<75$ & $\leq 60$ & $<70$ \\
\hline $3-6$ months & $<75$ & $\leq 80$ & $<70$ \\
\hline $6-12$ months & $<75$ & $\leq 80$ & $<70$ \\
\hline $1-2$ years & $<75$ & $\leq 90$ & $<72$ \\
\hline $2-3$ years & $<80$ & $\leq 90$ & $<74$ \\
\hline 3-4 years & $<80$ & $\leq 90$ & $<76$ \\
\hline 4-5 years & $<80$ & $\leq 90$ & $<78$ \\
\hline $5-6$ years & $<90$ & $\leq 90$ & $<80$ \\
\hline $6-7$ years & $<90$ & $\leq 90$ & $<82$ \\
\hline 7-8years & $<90$ & $\leq 90$ & $<84$ \\
\hline 8-9years & $<90$ & $\leq 90$ & $<86$ \\
\hline 9-10years & $<90$ & $\leq 90$ & $<88$ \\
\hline $10-12$ years & $<90$ & $\leq 90$ & $<90$ \\
\hline $12-13$ years & $<105$ & $\leq 100$ & $<90$ \\
\hline 13-14years & $<105$ & $\leq 100$ & $<90$ \\
\hline 14-16years & $<105$ & $\leq 100$ & $<90$ \\
\hline
\end{tabular}

APLS, Advanced Paediatric Life Support; PALS, Paediatric Advanced Life Support; PEWS, Paediatric Early Warning Score. (table 1). Heart rate was categorised as tachycardia versus no tachycardia according to the same reference as the BP cut-off (online supplementary appendix 1). Children with bradycardia $(5.9 \%-7.4 \%) \%)$ were defined as no tachycardia. Age was categorised as $0-1$ year, $1-2$ years, $2-5$ years, $5-12$ years and $12-16$ years. Triage urgency was determined by MTS V.3. ${ }^{22}$ Ill appearance was assessed by the nurse on a 2-point scale: ill versus nonill appearance.

\section{Data analysis}

Our sample was limited to patients with measured heart rate and BP. Children who died in the ED were excluded $(n=34)$. The value of BP measurement could be limited in this group, since the majority (94\%) was triaged as emergencies. Outliers were verified in patient records. First, we assessed the relation between BP and heart rate, using scatter plots. To facilitate analysis across age groups, we standardised heart rate and BP using z-scores, which were calculated separately for the different age categories. Second, we assessed the association between hypotension and serious illness using the three clinical cut-offs for hypotension. We used univariable logistic regression to evaluate the association of different BP cut-offs with ICU or with hospital admission, and adjusted for tachycardia in a multivariable model.

We determined the predictive value of hypotension for ICU admission and hospitalisation by calculating sensitivity, specificity, and positive and negative likelihood ratios. ${ }^{23}$ To study the predictive value of hypotension in addition to tachycardia, we calculated the predictive value of (1) hypotension; (2) tachycardia; (3) the combination of tachycardia and hypotension; 4) Either hypotension or tachycardia. Positive likelihood ratios $>5$ and negative likelihood ratios $<0.2$ were considered relevant. ${ }^{24}$

The normal range of Shock Index (ratio of heart rate to BP) is age dependent. ${ }^{25}$ Therefore, we stratified the analysis for Shock Index by age. To assess the association of Shock Index, we used univariable logistic regression. To facilitate interpretation, the OR present the odds for 0.1 unit increase in Shock Index. Next, the discriminative ability was presented by the area under the curve (AUC) of receiver operating characteristics. We used Youden's Index to identify the optimal cut-off value to assess the predictive value. ${ }^{26}$ We merged the age groups into $<2$ years, $2-10$ years and $>10$ years to ensure sufficient numbers for statistical analysis. To explore age-adjusted cut-off values for high Shock Index, we defined a cut-off by dividing the APLS tachycardia value with the APLS hypotension value for each age group (online supplementary appendix 2).

Subgroup analyses were performed in patients with ill appearance, fever (temperature $>38^{\circ} \mathrm{C}$ ) and patients presenting with surgical problems including major trauma, head injury, limb problems, wounds, torso injuries and assault. ${ }^{27}$

Data analyses and visualisation were performed in SPSS V.24.0 and $\mathrm{R}$. The medical ethical committee waived the requirement for informed consent.

\section{RESULTS}

During the study period, 45495 children $(58.6 \%$ male) presented to the $\mathrm{ED} ; 891$ (2.0\%) were triaged as emergencies. A total of 10698 patients had BP and heart rate measured. In this sample, $3907(36.5 \%)$ children were admitted to the general ward and $631(5.9 \%)$ were admitted to the ICU (table 2). Patients with BP measurement were older, had higher urgency level and were more often admitted compared with children without BP measurement (online supplementary appendix 3). 
Table 2 Characteristics of visits at the paediatric emergency department of Sophia Children's Hospital from 2009 to 2016

\begin{tabular}{|c|c|c|c|}
\hline & Total & $\begin{array}{l}\text { Patients with blood pressure and heart } \\
\text { rate measured }\end{array}$ & $\begin{array}{l}\text { Patients with hypotension according } \\
\text { to APLS }\end{array}$ \\
\hline & $n=45495$ & $n=10698$ & $\mathrm{n}=504$ \\
\hline Male; $\mathbf{n} \%$ & $26338(57.9)$ & $5872(54.9)$ & $219(43.5)$ \\
\hline Age in years; median, (IQR) & $4.3(1.4-9.8)$ & $7.74(3.6-7.7)$ & $13.0(6.67-14.5)$ \\
\hline \multicolumn{4}{|l|}{ Age category; n (\%) } \\
\hline $0-1$ year & $8734(19.2)$ & $920(8.6)$ & $78(15.5)$ \\
\hline $1-2$ years & $5736(12.6)$ & $668(6.2)$ & $7(1.4)$ \\
\hline $2-5$ years & $10154(22.3)$ & $2091(19.5)$ & $14(2.8)$ \\
\hline $5-12$ years & $13503(29.7)$ & $4101(38.3)$ & $80(15.9)$ \\
\hline $12-16$ years & $7368(16.2)$ & $2918(27.3)$ & $325(64.5)$ \\
\hline \multicolumn{4}{|l|}{ MTS urgency; $\mathbf{n}(\%)$} \\
\hline Emergent/very urgent & $6433(14.2)$ & $2572(24.0)$ & $155(30.7)$ \\
\hline Urgent & $19873(43.7)$ & $5026(47.0)$ & $199(39.5)$ \\
\hline Standard/non-urgent & $17711(38.9)$ & $2922(27.3)$ & $163(27.0)$ \\
\hline Missing & $1478(3.2)$ & $178(1.7)$ & $14(2.8)$ \\
\hline \multicolumn{4}{|l|}{ Disposition; $\mathbf{n}(\%)$} \\
\hline Admission general ward & 8848 (19.4) & 3276 (30.6) & $169(33.5)$ \\
\hline Intensive care & $1132(2.5)$ & $631(5.9)$ & $70(13.9)$ \\
\hline Died & $34(0.1)$ & $-{ }^{*}$ & $-^{*}$ \\
\hline Discharge & $34913(76.7)$ & $6719(62.8)$ & $261(51.8)$ \\
\hline Other & $401(0.9)$ & $61(0.6)$ & $4(0.8)$ \\
\hline Missing & $167(0.4)$ & $11(0.1)$ & $0(0.0)$ \\
\hline \multicolumn{4}{|l|}{ Shock Index; mean (SD) } \\
\hline $0-1$ year & & $1.52(0.48)$ & \\
\hline $1-2$ years & & $1.25(0.31)$ & \\
\hline $2-5$ years & & $1.11(0.26)$ & \\
\hline $5-12$ years & & $0.89(0.24)$ & \\
\hline $12-16$ years & & $0.76(0.22)$ & \\
\hline
\end{tabular}

*Children who died were excluded.

APLS, Advanced Paediatric Life Support; MTS, Manchester Triage System.

The prevalence of hypotension ranged from $1.2 \%$ to $5.3 \%$ depending on the cut-off used (online supplementary appendix 4). In children with hypotension according to APLS, $13.9 \%$ were admitted to the ICU and $33.5 \%$ were hospitalised.

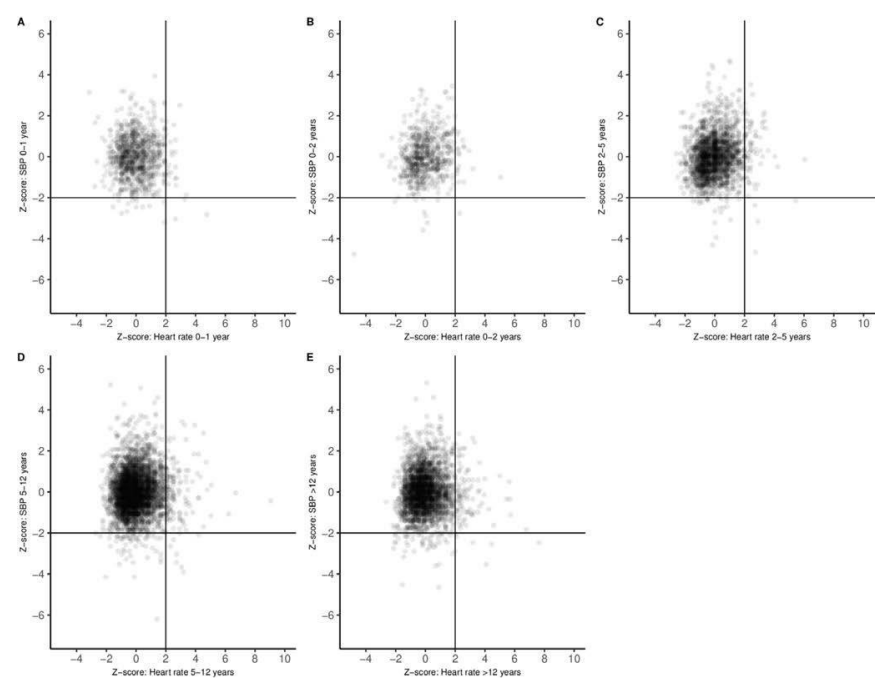

Figure 1 Scatter plots of z-scores of heart rate and systolic blood pressure (SBP) for different age categories $(A ; 0-1$ year, $B ; 1-2$ years, $C$; 2-5 years, $D ; 5-12$ years, E; $12-16$ years).
Our study found no association between z-scores of heart rate and BP in any of the age categories (Pearson correlation 0.040.18) (figure 1). In particular, no clear relation was observed between low BP and high z-scores for heart rate.

Hypotension, as a sole predictor, had an association with ICU admission (range OR 2.56-5.27) and hospital admission (range OR 1.4.66-2.66). The association between hypotension and serious illness remained significant after adjustment for tachycardia. In this analysis, the PALS cut-off for hypotension showed the strongest association with ICU admission and hospitalisation (table 3).

The cut-offs for hypotension showed a low sensitivity and a high specificity for serious illness (table 4). For ICU admission, specificity ranged between 0.95 and 0.99 and sensitivity between 0.05 and 0.12 . The positive likelihood ratios ranged from 2.38 to 5.06 and the negative likelihood ratios ranged from 0.93 to 0.96. The combination of tachycardia and hypotension did not improve the performance for ICU admission with low sensitivity (0.02-0.08) and high specificity (0.94-0.98). The analysis for hospital admission showed similar results.

Average values for Shock Index decreased with age. Stratified by age, Shock Index was associated with ICU admission (range OR 1.07-1.22) and hospitalisation (range OR 1.06-1.19) (table 3). The discriminative ability for Shock Index was poor for admission to ICU (range AUC 0.59-0.63) or admission to the hospital (range AUC 0.58-0.62) (online supplementary appendix 5 ). The identified cut-offs per age group had low sensitivity 
Table 3 Logistic regression analysis for ICU and hospital admission

\begin{tabular}{|c|c|c|c|c|c|}
\hline & \multirow[t]{2}{*}{$\begin{array}{l}\text { Patients with } \\
\text { hypotension/tachycardia }\end{array}$} & \multicolumn{2}{|c|}{ ICU admission } & \multicolumn{2}{|c|}{ Hospital admission } \\
\hline & & OR & $95 \% \mathrm{Cl}$ & $\mathrm{OR}$ & $95 \% \mathrm{Cl}$ \\
\hline \multicolumn{6}{|l|}{ APLS $^{18}$} \\
\hline Hypotension & $\mathrm{n}=504$ & 2.77 & 2.12 to 3.62 & 1.61 & 1.34 to 1.92 \\
\hline Tachycardia (APLS) & $n=1692$ & 2.46 & 2.06 to 2.94 & 2.62 & 2.36 to 2.91 \\
\hline Hypotension adjusted for tachycardia & & 2.68 & 2.05 to 3.51 & 1.56 & 1.30 to 1.88 \\
\hline \multicolumn{6}{|l|}{ PALS/septic shock screening tool ${ }^{1920}$} \\
\hline Tachycardia (septic shock screening tool) & $n=1709$ & 1.80 & 1.49 to 2.18 & 1.91 & 1.72 to 2.12 \\
\hline Hypotension adjusted for tachycardia & & 4.99 & 3.32 to 7.52 & 2.52 & 1.77 to 3.59 \\
\hline \multicolumn{6}{|l|}{ PEWS $^{34}$} \\
\hline Hypotension & $\mathrm{n}=571$ & 2.56 & 1.98 to 3.31 & 1.46 & 1.24 to 1.73 \\
\hline Tachycardia (PEWS) & $n=4113$ & 2.02 & 1.72 to 2.37 & 2.16 & 1.99 to 2.34 \\
\hline Age $2-5$ years & & 1.08 & 1.02 to 1.15 & 1.06 & 1.02 to 1.09 \\
\hline Age $5-12$ years & & 1.13 & 1.08 to 1.19 & 1.14 & 1.11 to 1.18 \\
\hline Age $>12$ years & & 1.22 & 1.15 to 1.29 & 1.19 & 1.15 to 1.24 \\
\hline
\end{tabular}

${ }^{*}$ ORs present each 0.1 increase in Shock Index.

APLS, Advanced Paediatric Life Support; ICU, intensive care unit; PALS, Paediatric Advanced Life Support; PEWS, Paediatric Early Warning Score.

(range 0.27-0.42) and moderate specificity (range 0.79-0.91) for ICU admission. None of the identified Shock Index cut-offs had acceptable positive or negative likelihood ratios (online supplementary appendix 6).

The APLS Shock Index cut-off performed similarly with low sensitivity and high specificity (online supplementary appendix 7). The positive likelihood ratio was 3.86 (95\% CI 3.1 to 4.8 ) and negative likelihood ratio was 0.89 (95\% CI 0.87 to 0.92 ).

In febrile children, patients with ill appearance and surgical patients, the hypotension and Shock Index cut-offs showed similar performance. For Shock Index, the highest AUC was found for febrile patients aged $>10$ years for ICU admission (0.75 95\% CI 0.63 to 0.87 ) (online supplementary appendix 8).

\section{DISCUSSION}

In our observational cohort, hypotension has a significant association with serious illness when corrected for tachycardia. However, hypotension showed low sensitivity and high specificity for serious illness in children with routinely measured BP in the ED. The combination of hypotension and tachycardia did not improve the sensitivity further. In addition, although Shock Index was associated with serious illness, acceptable cut-off values could not be identified for different age groups.

Accurate reference values for abnormal vital signs are essential to avoid misclassification. Values based on healthy children may not be accurate for children in the ED, as ill children may present with pain and distress which influences heart rate and BP values. Expert-based cut-offs for low BP are currently used. However, these are not based on large studies and show large variation and are therefore not a good alternative. For example, more than $50 \%$ of the children with hypotension according to the APLS were discharged home following ED visit. Two recent studies presented BP reference ranges and distributions for critically ill children but validated reference values for the paediatric ED population are lacking. ${ }^{28} 29$
Hypotension is considered a late sign of illness that is preceded by an increase in heart rate. To preserve cardiac output, children compensate by elevating heart rate and systemic vascular resistance. When this compensatory mechanism is inadequate, BP could drop which may indicate shock. ${ }^{7}$ Our study showed that heart rate and BP were not correlated. In particular, high z-scores of heart rate did not correlate with low z-scores of BP. Moreover, irrespective of tachycardia, cutoffs for hypotension showed a significant association with serious illness.

We focused on tachycardia as this is an early indicator of critical illness and these children could benefit from measuring BP. Bradycardia, however, indicates irreversible shock. Seriously ill children with bradycardia present with lack of perfusion resulting in cardiopulmonary arrest. ${ }^{30}$ Therefore, BP measurement could have limited additional value in children with bradycardia. Furthermore, we did not analyse other predictors of serious illness. In practice, however, heart rate and BP are evaluated with other clinical markers which can be more sensitive predictors for serious illness. Future studies should focus on the combination of $\mathrm{BP}$ and other clinical predictors to evaluate the additional value of $\mathrm{BP}$ in practice.

Shock Index is associated with mortality in children with septic shock. ${ }^{12} 13$ Research on Shock Index in EDs has mainly focused on injured patients. ${ }^{14}{ }^{15}$ No reference values exist for the whole age range in children. Acker et al proposed age-adjusted cut-offs according to normal vital signs for children $>4$ years. However, a recent study showed that $2.3 \%$ of healthy children had abnormal values according to this definition. ${ }^{6}$ Our study found an association between Shock Index and serious illness in different age groups. For children $>12$ years a 0.1 unit increase in Shock Index relates to odds of 1.22 for ICU admission. However, the discriminative ability for Shock Index was poor. In general, neither of the identified cut-off values had both acceptable sensitivity and specificity. 


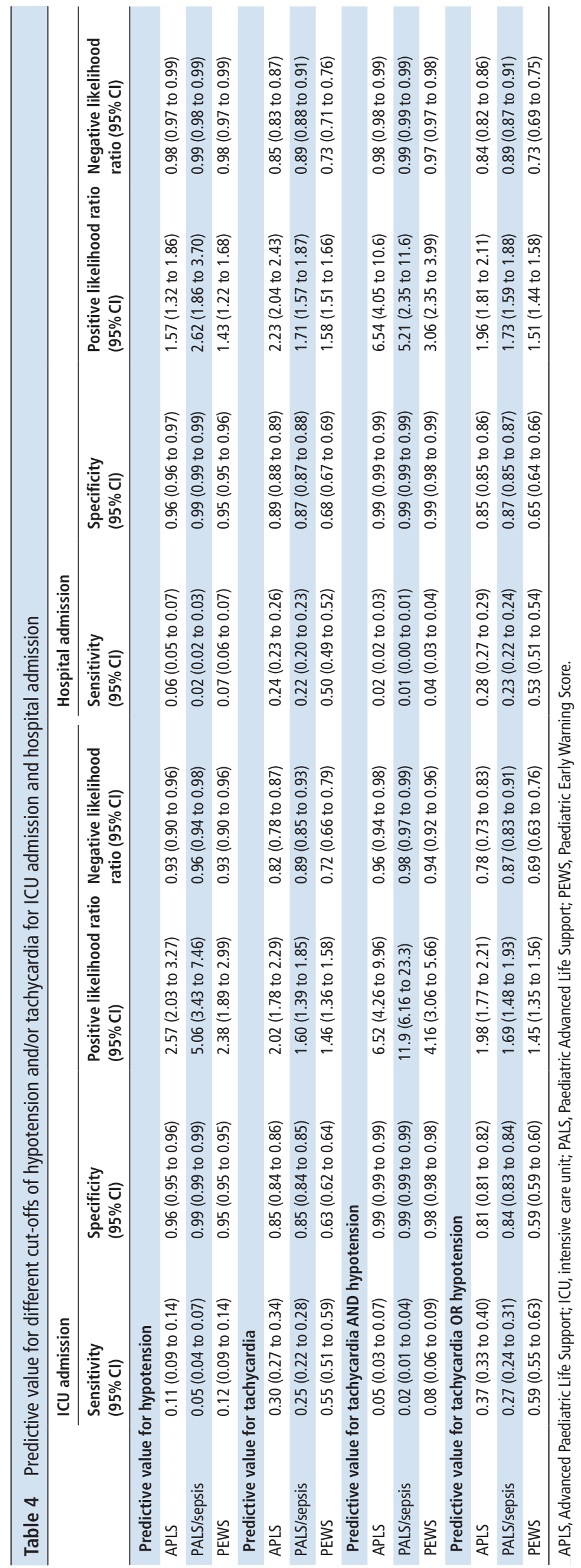


We focused our analysis on high Shock Index values to detect severe illness. We acknowledge that low Shock Index values are also abnormal. Due to the vasopressor response, patients with increased intracranial pressure will have low heart rate and high BP leading to low Shock Index values.

Although hypotension showed high specificity for serious illness, the sensitivity was very low, regardless of the used definition. The combination of hypotension and tachycardia did not improve the sensitivity or the specificity for predicting serious illness. PALS ${ }^{19}$ had good rule-in value having good specificity and high positive likelihood ratios. However, for early recognition of severely ill children in the ED, it is important to rule out serious illness. Hypotension and tachycardia lack these characteristics, having low sensitivity and poor negative likelihood ratios for serious illness. Considering that accurate BP measurement is time-consuming for nurses, ${ }^{31}$ these results suggest limited value of routine BP measurement in all children attending the ED.

Strengths of this study are the use of three hypotension cut-offs that are widely used in clinical practice. In addition, our analyses were based on a large cohort of paediatric ED patients of all ages with different presenting problems. We used routine data and therefore our results are representative of clinical practice.

This study has some limitations. First, patients were included when BP and heart rate were measured. This selected group is more severely ill, comprising older children, more highly urgent cases and more ICU admissions. This could potentially bias our findings. However, this reflects measurement of $\mathrm{BP}$ in the practice of the ED. The frequency of BP investigation and the increase with age and urgency was similar to previous studies. ${ }^{12}$ In addition, the population of our tertiary university hospital consists of more children with comorbidities and more severely ill children. In settings with low prevalence of serious illness, less yield could be expected. Second, we used hospital admission and ICU admission to define serious illness. These outcomes are widely used in literature and are applicable to large data sets. ${ }^{21} 3233$ As reasons for ICU admission following ED visit include lifethreatening conditions, the presence of hypotension could have influenced the decision for ICU admission. Hospital admission could occur for various conditions as fractures or bronchiolitis which are unlikely to develop low BP. Furthermore, accurate measurement of BP in children in the ED is challenging. Movement of limbs and uncooperativeness interfere with the measurements. Moreover, the correct cuff size and technique need to be applied. Therefore, the quality of BP measurement should be taken into account.

Finally, our study aimed to evaluate the value of routine BP measurements in children for the recognition of serious illness. We acknowledge that BP measurement may be indicated in the ED for diagnostics, detection of hypertension, follow-up or therapy monitoring.

\section{CONCLUSION}

Our observational study demonstrates that hypotension is associated with serious illness, independent of heart rate. Although the specificity of hypotension is high, the sensitivity for serious illness is very low. The combination of hypotension and tachycardia did not further improve the sensitivity. Shock Index is related to serious illness, however we could not identify acceptable cut-off values. These findings suggest limited value of measuring routine $\mathrm{BP}$ to detect hypotension in all attending children. Future studies need to investigate which specific patients could benefit from BP measurement and should focus on developing accurate reference values for hypotension and Shock Index that are applicable in the ED.

Correction notice This article has been corrected since it first published online. The open access licence type has been amended.

Contributors All authors have read and approved the final version of the manuscript. All authors substantially contributed to the writing (ie, drafting and/or critical revision) of the manuscript.

Funding The authors have not declared a specific grant for this research from any funding agency in the public, commercial or not-for-profit sectors.

Competing interests None declared.

Patient consent for publication Not required.

Ethics approval The medical ethical committee approved the study.

Provenance and peer review Not commissioned; externally peer reviewed.

Data sharing statement No additional data are available.

Open access This is an open access article distributed in accordance with the Creative Commons Attribution 4.0 Unported (CC BY 4.0) license, which permits others to copy, redistribute, remix, transform and build upon this work for any purpose, provided the original work is properly cited, a link to the licence is given, and indication of whether changes were made. See: https://creativecommons.org/ licenses/by/4.0/.

\section{ORCID ID}

Nienke N Hagedoorn http://orcid.org/0000-0001-9237-4904

\section{REFERENCES}

1 Gilhotra Y, Willis F. Blood pressure measurements on children in the emergency department. Emerg Med Australas 2006;18:148-54.

2 Silverman MA, Walker AR, Nicolaou DD, et al. The frequency of blood pressure measurements in children in four EDs. Am J Emerg Med 2000;18:784-8.

3 Thompson T, Stanford K, Dick R, et al. Triage assessment in pediatric emergency departments: a national survey. Pediatr Emerg Care 2010;26:544-8.

4 Haque IU, Zaritsky AL. Analysis of the evidence for the lower limit of systolic and mean arterial pressure in children. Pediatr Crit Care Med 2007;8:138-44.

5 Marlais M, Lyttle MD, Inwald D. Ten concerns about blood pressure measurement and targets in paediatric sepsis. Intensive Care Med 2017;43:433-5.

6 Sarganas G, Schaffrath Rosario A, Berger S, et al. An unambiguous definition of pediatric hypotension is still lacking: Gaps between two percentile-based definitions and Pediatric Advanced Life Support/Advanced Trauma Life Support guidelines. J Trauma Acute Care Surg 2019;86:448-453.

7 Brierley J, Carcillo JA, Choong K, et al. Clinical practice parameters for hemodynamic support of pediatric and neonatal septic shock: 2007 update from the American College of Critical Care Medicine. Crit Care Med 2009;37:666-88.

8 Dellinger RP, Levy MM, Rhodes A, et al. Surviving sepsis campaign: international guidelines for management of severe sepsis and septic shock: 2012. Crit Care Med 2013;41:580-637.

9 Rady MY, Smithline HA, Blake H, et al. A comparison of the shock index and conventional vital signs to identify acute, critical illness in the emergency department. Ann Emerg Med 1994;24:685-90.

10 Wira CR, Francis MW, Bhat S, et al. The shock index as a predictor of vasopressor use in emergency department patients with severe sepsis. West J Emerg Med 2014;15:60-6.

11 Balhara KS, Hsieh YH, Hamade B, et al. Clinical metrics in emergency medicine: the shock index and the probability of hospital admission and inpatient mortality. Emerg Med J 2017;34:89-94.

12 Yasaka Y, Khemani RG, Markovitz BP. Is shock index associated with outcome in children with sepsis/septic shock?*. Pediatr Crit Care Med 2013;14:e372-9.

13 Rousseaux J, Grandbastien B, Dorkenoo A, et al. Prognostic value of shock index in children with septic shock. Pediatr Emerg Care 2013;29:1055-9.

14 Acker SN, Bredbeck B, Partrick DA, et al. Shock index, pediatric age-adjusted (SIPA) is more accurate than age-adjusted hypotension for trauma team activation. Surgery 2017;161:803-7.

15 Linnaus ME, Notrica DM, Langlais CS, et al. Prospective validation of the shock index pediatric-adjusted (SIPA) in blunt liver and spleen trauma: An ATOMAC+ study. J Pediatr Surg 2017; 52:340-4.

16 Zachariasse JM, Seiger N, Rood PP, et al. Validity of the Manchester Triage system in emergency care: a prospective observational study. PLoS One 2017;12:e0170811.

17 Zachariasse JM, Kuiper JW, de Hoog M, et al. Safety of the Manchester Triage System to Detect Critically III Children at the Emergency Department. J Pediatr 2016;177:232-7

18 Turner NM, Leroy PL. Advanced paediatric life support. 5th ed: Bohn Stafleu van Loghum, 2017. 
19 Kleinman ME, Chameides L, Schexnayder SM, et al. Pediatric advanced life support: 2010 American Heart Association Guidelines for Cardiopulmonary Resuscitation and Emergency Cardiovascular Care. Pediatrics 2010;126:e1361-99.

20 Davis AL, Carcillo JA, Aneja RK, et al. American college of critical care medicine clinical practice parameters for hemodynamic support of pediatric and neonatal septic shock. Crit Care Med 2017:45:1061-93.

21 Parshuram CS, Dryden-Palmer K, Farrell C, et al. Effect of a pediatric early warning system on all-cause mortality in hospitalized pediatric patients: the EPOCH randomized clinical trial. JAMA 2018;319:1002-12.

22 van Veen M, Steyerberg EW, Van't Klooster M, et al. The Manchester triage system: improvements for paediatric emergency care. Emerg Med J 2012;29:654-9.

23 Herbert R. Confidence Interval Calculator, 2013 https://www.pedro.org.au/english/ downloads/confidence-interval-calculator/ Accessed on 01-10-2017.

24 Grimes DA, Schulz KF. Refining clinical diagnosis with likelihood ratios. Lancet 2005;365:1500-5.

25 Rappaport LD, Deakyne S, Carcillo JA, et al. Age- and sex-specific normal values for shock index in National Health and Nutrition Examination Survey 1999-2008 for ages 8 years and older. Am J Emerg Med 2013;31:838-42.

26 Youden WJ. Index for rating diagnostic tests. Cancer 1950;3:32-5.
27 van Veen M, Steyerberg EW, Ruige M, et al. Manchester triage system in paediatric emergency care: prospective observational study. BMJ 2008;337:a1501.

28 Eytan D, Goodwin AJ, Greer R, et al. Heart rate and blood pressure centile curves and distributions by age of hospitalized critically ill children. Front Pediatr 2017;5:52.

29 Abdelrazeq S, Ray S, Rogers L, et al. Age-associated blood pressure distributions in paediatric intensive care units differ from healthy children. Intensive Care Med 2018;44:384-6.

30 Wang EN, Chan C, Anand S, et al. Considerations in the Management of Shock in the Pediatric Trauma Patient. Pediatric Emergency Medicine Reports 2006:11.

31 Poor KM, Ducklow TB. Benefit of BP Measurement in Pediatric ED Patients. ISRN Nurs 2012;2012:1-6.

32 Chaiyakulsil C, Pandee U. Validation of pediatric early warning score in pediatric emergency department. Pediatr Int 2015;57:694-8.

33 Gold DL, Mihalov LK, Cohen DM. Evaluating the Pediatric Early Warning Score (PEWS) system for admitted patients in the pediatric emergency department. Acad Emerg Med 2014;21:1249-56.

34 Parshuram CS, Duncan HP, Joffe AR, et al. Multicentre validation of the bedside paediatric early warning system score: a severity of illness score to detect evolving critical illness in hospitalised children. Crit Care 2011:15:R184. 